ULB-TH-00-29

UNIL-IPT-00-27

\title{
Three generations on a local vortex in extra dimensions.
}

\author{
J.-M.Frère ${ }^{1}$, M.V.Libanov ${ }^{2,1}$ and S.V.Troitsky ${ }^{2,3,1}$ \\ 1 Service de Physique Théorique, CP 225, \\ Université Libre de Bruxelles, B-1050, Brussels, Belgium; \\ 2 Institute for Nuclear Research of the Russian Academy of Sciences, \\ 60th October Anniversary Prospect 7a, 117312, Moscow, Russia; \\ 3 Institute of Theoretical Physics, University of Lausanne, \\ CH-1015, Lausanne, Switzerland
}

\begin{abstract}
We develop an approach to the origin of three generations of the Standard Model fermions from one generation in a higher-dimensional theory, where fourdimensional fermions appear as zero modes trapped in the core of a topological defect, and the hierarchy of masses and mixings is produced by wave function overlaps in extra dimensions. We present a model with unbroken $U(1)$ symmetry where three zero modes appear on an Abrikosov-Nielsen-Olesen vortex due to nontrivial scalar-fermion interactions.
\end{abstract}


1. Models of particle physics in more than four spacetime dimensions, where the Standard Model fields are localized on a four-dimensional topological defect, or "brane", open new possibilities to explain the misterious pattern of fermion mass hierarchies, as well as the origin of three generations of fermions with identical quantum numbers. If different fermionic modes have different wave function profiles in extra dimensions, then their overlaps with the Higgs wave function may produce hierarchical structure of masses and mixings [1]. Of particular interest are the class of models [2] where each multi-dimensional fermion develops three chiral zero modes localized on a four-dimensional brane. This occurs due to certain topological properties of the brane background. The Index theorem guarantees that the three zero modes are linearly independent, and thus have different profiles in extra dimensions. Analysis of the equations for these zero modes demonstrates that a hierarchy in the mass matrix indeed appears due to overlaps of the wave functions. For the discussion of this mechanism and comparison with other approaches, see Ref. [2].

The main drawback of the models of Ref. [2] is the complications needed to generate nonzero inter-generation mixings. In a particular model, it was necessary to break explicitly a $U(1)$ symmetry, which gave rise to nontrivial topology of the vortex (which played the role of a brane). With unbroken $U(1)$, the mixings were zero. Explicitly broken $U(1)$ could not be gauge symmetry, thus the vortex had to be global and had to have logarithmically divergent energy. Furthermore, the question whether the vortex is stable with broken $U(1)$ remained open.

In this Letter, we discuss an improved version of the models of Ref. [2]. We present a model, in which a similar mechanism gives rise to three chiral fermionic generations on a vortex embedded in a six-dimensional spacetime, with hierarchical pattern of masses and mixing angles, but the corresponding $U(1)$ symmetry is unbroken. In this way we overcome the problems mentioned above. The cost for unbroken $U(1)$ is the necessity to invoke operators of higher dimensionality in the scalar-fermion interactions. We include, however, all lowest dimensionality operators consistent with the gauge symmetries of the Standard Model and the gauge $U(1)$ symmetry which gives rise to nontrivial topology of the vortex. The requirement of renormalizability of the theory does not make much sense anyway in the six-dimensional models, since even usual Yukawa scalar-fermion-fermion coupling is non-renormalizable. Thus, any 


\begin{tabular}{|c|c|c|c|c|c|}
\hline \multirow[t]{2}{*}{ fields } & \multirow[t]{2}{*}{ profiles } & \multicolumn{2}{|c|}{ charges } & \multicolumn{2}{|c|}{ representations } \\
\hline & & $U(1)_{g}$ & $U(1)_{Y}$ & $S U(2)_{W}$ & $S U(3)_{C}$ \\
\hline scalar $\Phi$ & $\begin{array}{c}F(r) \mathrm{e}^{i \theta} \\
F(0)=0, F(\infty)=v_{\Phi}\end{array}$ & +1 & 0 & 1 & 1 \\
\hline scalar $X$ & $\begin{array}{c}X(r) \\
X(0)=v_{X}, X(\infty)=0\end{array}$ & +1 & 0 & 1 & 1 \\
\hline scalar $H$ & $\begin{array}{c}H(r) \\
H(0)=v_{H}, H(\infty)=0\end{array}$ & -1 & $+1 / 2$ & 2 & 1 \\
\hline fermion $Q$ & $3 \mathrm{~L}$ zero modes & axial $+3 / 2$ & $+1 / 6$ & 2 & 3 \\
\hline fermion $U$ & $3 \mathrm{R}$ zero modes & axial $-3 / 2$ & $+2 / 3$ & 1 & 3 \\
\hline fermion $\quad D$ & $3 \mathrm{R}$ zero modes & axial $-3 / 2$ & $-1 / 3$ & 1 & 3 \\
\hline fermion $L$ & $3 \mathrm{~L}$ zero modes & axial $+3 / 2$ & $-1 / 2$ & 2 & 1 \\
\hline fermion & $3 \mathrm{R}$ zero modes & axial $-3 / 2$ & -1 & 1 & 1 \\
\hline
\end{tabular}

Table 1: Scalars and fermions with their gauge quantum numbers. For convenience, we describe here also the profiles of the classical scalar fields and fermionic wave functions in extra dimensions.

multidimensional field theory is usually considered as a low energy limit of some more fundamental model which includes gravity as well.

2. The matter field content of the model is summarized in Table 1. The scalar field $\Phi$, together with $U(1)_{g}$ gauge field, forms a vortex, while two other scalars, $X$ and $H$, develop profiles localized on the vortex. There is one fermionic generation which consists of five six-dimensional fermions $Q, U, D, L$, and $E$. Each of the fermions develop, in the vortex background, three chiral zero modes localized in the core of the vortex, which correspond to three generations of the Standard Model fermions. We do not discuss here subtle issues of localizing gauge fields on the four-dimensional brane.

We use the notations of Ref. [2]; in particular, we denote four-dimensional indices by Greek letters, $x_{\mu}, \mu=0, \ldots, 3$, and introduce polar coordinates $r, \theta$ in the $x_{4}, x_{5}$ plane. 
Let us consider the following scalar potential:

$$
V_{s}=\frac{\lambda}{2}\left(|\Phi|^{2}-v^{2}\right)^{2}+\frac{\kappa}{2}\left(|H|^{2}-\mu^{2}\right)^{2}+h^{2}|H|^{2}|\Phi|^{2}+\frac{\rho}{2}\left(|X|^{2}-v_{1}^{2}\right)^{2}+\eta^{2}|X|^{2}|\Phi|^{2} .
$$

We study solutions to the classical field equations for the system involving three scalar fields $\Phi, X$ and $H$ as well as the $U(1)_{g}$ gauge field. Let us restrict ourselves to topologically nontrivial solutions to the classical field equations which do not depend on $x_{\mu}$. For a certain range of parameters, the lowest energy solution of this kind is given by a vortex configuration build up of the gauge field and $\Phi$, where

$$
\Phi=\mathrm{e}^{i \theta} F(r)
$$

Two other scalar fields do not depend on $\theta$, are nonzero at $r=0$ and satisfy the following boundary conditions:

$$
\left.\frac{d X}{d r}\right|_{r=0}=\left.\frac{d H}{d r}\right|_{r=0}=0, \quad X(r=\infty)=H(r=\infty)=0 .
$$

This solution describes a superconducting bosonic string [3] with currents of two different fields, $H$ and $X$. Far from the core of the string, $H(r)$ and $X(r)$ fall off exponentially, so the two scalar fields are localized close to the four-dimensional hypersurface $r=0$.

For the scalar-fermion interactions, we take the most general operators of the lowest order, consistent with gauge invariance,

$$
\begin{gathered}
V_{s f}=V_{1}+V_{2}+V_{3}, \\
V_{1}=g_{q} \Phi^{3} \bar{Q} \frac{1-\Gamma_{7}}{2} Q+g_{u} \Phi^{* 3} \bar{U} \frac{1-\Gamma_{7}}{2} U+g_{d} \Phi^{* 3} \bar{D} \frac{1-\Gamma_{7}}{2} D+ \\
g_{l} \Phi^{3} \bar{L} \frac{1-\Gamma_{7}}{2} L+g_{e} \Phi^{* 3} \bar{E} \frac{1-\Gamma_{7}}{2} E+\text { h.c. } \\
V_{2}=Y_{d} H X \bar{Q} \frac{1-\Gamma_{7}}{2} D+Y_{u} \tilde{H} X^{*} \bar{Q} \frac{1-\Gamma_{7}}{2} U+Y_{l} H X \bar{L} \frac{1-\Gamma_{7}}{2} E+\text { h.c. } \\
V_{3}=Y_{d} \epsilon_{d} H \Phi \bar{Q} \frac{1-\Gamma_{7}}{2} D+Y_{u} \epsilon_{u} \tilde{H} \Phi^{*} \bar{Q} \frac{1-\Gamma_{7}}{2} U+Y_{l} \epsilon_{l} H \Phi \bar{L} \frac{1-\Gamma_{7}}{2} E+\text { h.c. }
\end{gathered}
$$

where $\tilde{H}_{i}=\epsilon_{i j} H_{j}^{*}, i, j$ are $S U(2)_{W}$ indices (we denote Yukawa coupling constants in Eq. (2) as $Y_{u, d, l}$, and in Eq. (3) as $Y_{u} \epsilon_{u}, \ldots$, for convenience).

Note that the scalar background $\Phi^{3}$, where the field $\Phi$ has the winding number one, $\Phi=F(r) \mathrm{e}^{i \theta}$, has exactly the same topological properties as the background $\Phi_{1}$ 
of the vortex with winding number three, $\Phi_{1}=F_{1}(r) \mathrm{e}^{3 i \theta}$. As a result, the interactions Eq. (1) provide three left-handed (right-handed) zero modes for each of the fermions $Q, L(U, D, E)$. All of these modes are localized in the core of the vortex. These modes were discussed in detail in Ref. [2]; up to the four-dimensional plane waves they are:

$$
Q \sim \sum_{a}\left(\begin{array}{c}
0 \\
q_{3-a}(r) \mathrm{e}^{i(3-a) \theta} \\
q_{a-1}(r) \mathrm{e}^{-i(a-1) \theta} \\
0
\end{array}\right), \quad D \sim \sum_{a}\left(\begin{array}{c}
d_{a-1}(r) \mathrm{e}^{-i(a-1) \theta} \\
0 \\
0 \\
d_{3-a}(r) \mathrm{e}^{i(3-a) \theta}
\end{array}\right)
$$

where $a=1,2,3$ enumerates three modes (three fermionic generations), and each element of the spinor corresponds to a column of two elements, so that the spinors are eight-component. Modes for other fermions have the similar form with replacement $Q \rightarrow L, q \rightarrow l ; D \rightarrow U, d \rightarrow u ; D \rightarrow E, d \rightarrow e$. The radial functions have the following leading behaviour:

$$
f_{p}(r) \sim r^{p}, r \rightarrow 0 ; \quad f_{p}(r) \sim \mathrm{e}^{-g_{f} v r}, r \rightarrow \infty
$$

where $f$ stands for $q, u, d, l$, and $e$.

3. Masses of the four-dimensional fermions are generated by means of the interactions (2), (3). As we will see immediately, the terms in $V_{2}$, Eq. (2), are responsible for the diagonal mass terms, while $V_{3}$, Eq. (3), generates mixings. The field $X$ carries exactly the same gauge quantum numbers as $\Phi$ and is necessary to keep terms in both $V_{2}$ and $V_{3}$ gauge invariant.

To obtain the effective four-dimensional mass matrix, one has to perform the integration over extra dimensions. Let us discuss first the mass matrix of the down type quarks $(d, s, b)$. From the corresponding terms in Eq. (2) one gets the mass matrix elements

$$
m_{a b}^{d(1)}=Y_{d} \int r d r d \theta H(r) X(r) q_{3-a}(r) d_{3-b}(r) \mathrm{e}^{i(a-b) \theta}=m_{a a}^{d} \delta_{a b}
$$

Eq. (3) produces the contribution

$$
m_{a b}^{d(2)}=Y_{d} \epsilon_{d} \int r d r d \theta H(r) F(r) q_{3-a}(r) d_{3-b}(r) \mathrm{e}^{i(1+a-b) \theta}=m_{a, a+1}^{d} \delta_{a+1, b} .
$$


We see that the selection rules coming from $\theta$-dependence of the way functions restrict the mass matrix to the form

$$
m^{d}=\left(\begin{array}{ccc}
m_{11}^{d} & m_{12}^{d} & 0 \\
0 & m_{22}^{d} & m_{23}^{d} \\
0 & 0 & m_{33}^{d}
\end{array}\right)
$$

As was discussed in Ref. [2], the hierarchical pattern of masses is reproduced when the profile of the Higgs field $H$ in extra dimensions is narrow $\left(h \gg g_{f}, h \gg \sqrt{\lambda}\right)$. In this case, one can estimate, to the leading order, the elements of the mass matrix as

$$
\begin{aligned}
m_{a a}^{d} & =2 \pi Y_{d} \int r d r H(r) X(r) q_{3-a}(r) d_{3-a}(r) \sim\left(\sigma_{q} \sigma_{d}\right)^{(3-a)}, \\
m_{a, a+1}^{d} & =2 \pi Y_{d} \epsilon_{d} \int r d r H(r) F(r) q_{3-a}(r) d_{2-a}(r) \sim \bar{\lambda} \sigma_{q}^{3-a} \sigma_{d}^{2-a},
\end{aligned}
$$

in terms of small parameters $\sigma_{d}=g_{d} / h, \bar{\lambda}=\sqrt{\lambda} / h$. Hence,

$$
m^{d} \sim Y_{d}\left(\begin{array}{ccc}
\sigma_{q}^{2} \sigma_{d}^{2} & \epsilon_{d} \sigma_{q}^{2} \sigma_{d} \bar{\lambda} & 0 \\
0 & \sigma_{q} \sigma_{d} & \epsilon_{d} \bar{\lambda} \sigma_{q} \\
0 & 0 & 1
\end{array}\right)
$$

In a similar way, one obtains the mass matrices for up-type quarks $(u, c, t)$ and charged leptons $(e, \mu, \tau)$,

$$
m^{u} \sim Y_{u}\left(\begin{array}{ccc}
\sigma_{q}^{2} \sigma_{u}^{2} & 0 & 0 \\
\epsilon_{u} \sigma_{q} \sigma_{u}^{2} \bar{\lambda} & \sigma_{q} \sigma_{u} & 0 \\
0 & \epsilon_{u} \bar{\lambda} \sigma_{u} & 1
\end{array}\right), \quad m^{l} \sim Y_{l}\left(\begin{array}{ccc}
\sigma_{l}^{2} \sigma_{e}^{2} & \epsilon_{l} \sigma_{l}^{2} \sigma_{e} \bar{\lambda} & 0 \\
0 & \sigma_{l} \sigma_{e} & \epsilon_{l} \bar{\lambda} \sigma_{l} \\
0 & 0 & 1
\end{array}\right)
$$

These mass matrices correspond to Cabibbo-Kobayashi-Maskawa (CKM) mixing matrix of the form:

$$
U^{C K M} \simeq\left(\begin{array}{ccc}
1-\frac{1}{2} \zeta_{d}^{2} & \zeta_{d}-\mathrm{e}^{-i \phi} \zeta_{u} & -\zeta_{d} \zeta_{u} \mathrm{e}^{-i \phi} \\
\mathrm{e}^{i \phi} \zeta_{u}-\zeta_{d} & 1-\zeta_{d}^{2}+2 \cos \phi \zeta_{d} \zeta_{u} & \zeta_{d}-\mathrm{e}^{-i \phi} \zeta_{u} \\
\zeta_{d}^{2}-\mathrm{e}^{i \phi} \zeta_{u} \zeta_{d} & \zeta_{u}-\mathrm{e}^{i \phi} \zeta_{d} & 1-\frac{1}{2} \zeta_{d}^{2}+\mathrm{e}^{i \phi} \zeta_{d} \zeta_{u}
\end{array}\right)
$$

where $\zeta_{d} \sim\left|\epsilon_{d}\right| \bar{\lambda} \sigma_{q}, \zeta_{u} \sim\left|\epsilon_{u}\right| \bar{\lambda} \sigma_{q} \sigma_{u}^{2}$, and $\phi=\arg \epsilon_{u}$ (all but one complex phases of the parameters are unphysical)円.

${ }^{*}$ Note that $\zeta_{d}$ and $\zeta_{u}$ are of different order in $\sigma$; this reflects the fact that the symmetry of mixing matrices is not $u_{L} \leftrightarrow d_{L}$, but $u_{L} \leftrightarrow d_{R}$. 
As follows from Eqs. (4), (5), and (6), to the leading approximations, masses of nine charged fermions of the Standard Model are determined by six parameters: the overall mass scale, say, $Y_{d} v_{H} v_{X}$; two ratios of six-dimensional Yukawa couplings, $Y_{u} / Y_{d}$ and $Y_{l} / Y_{d}$; and three combinations $\left(\sigma_{q} \sigma_{u}\right),\left(\sigma_{q} \sigma_{d}\right),\left(\sigma_{l} \sigma_{e}\right)$. Four independent parameters of CKM matrix are determined by $\zeta_{u}, \zeta_{d}$, and $\phi$.

4. To conclude, we presented a mechanism to obtain three fermionic generations together with their hierarchical masses and mixing angles, from a single generation in a higher-dimensional theory. This mechanism is developed along the lines of Ref. [2] but does not require explicit breaking of the symmetry which gives rise to nontrivial topology of the brane. Due to higher-order interactions between scalar field and fermions, three zero modes are localized on a defect with topological number one.

In our particular model, the fermions are localized on an Abrikosov-NielsenOlesen vortex. With obvious modifications, the mechanism works for other topological defects, such as a global vortex, a monopole, or a hedgehod. The mechanism presented above can be embedded either in a theory with large extra dimensions [4] or in a model where gravity is localized on a topological defect [5].

M.L. and S.T. thank Université Libre de Bruxelles for kind hospitality. This work was partially supported by the "Actions de Recherche Concretées" of "Communauté Française de Belgique" and IISN-Belgium. The work of M.L. and S.T. is supported in part by RFFI grant 99-02-18410a, by CRDF award RP1-2103, by the Russian Academy of Sciences, JRP grant No. 37, by the Council for Presidential Grants and State Support of Leading Scientific Schools, grant 00-15-96626, and by the programme SCOPES of the Swiss National Science Foundation, project No. 7SUPJ062239, financed by Federal Department of Foreign affairs. The work of S.T. is supported in part by Swiss Science Foundation, grant 21-58947.99.

\section{References}

[1] N. Arkani-Hamed and M. Schmaltz, Phys. Rev. D61 (2000) 033005 thepph/9903417; G. Dvali and M. Shifman, Phys. Lett. B475 (2000) 295 hepph/0001072; T. Gherghetta and A. Pomarol, hep-ph/0003129; D. E. Kaplan 
and T. M. Tait, JHEP 0006 (2000) 020 [hep-ph/0004200; S. J. Huber and Q. Shafi, hep-ph/0010195.

[2] M. V. Libanov and S. V. Troitsky, hep-ph/0011095.

[3] E. Witten, Nucl. Phys. B249 (1985) 557.

[4] N. Arkani-Hamed, S. Dimopoulos, G. Dvali and J. March-Russell, hep-ph/9811448.

[5] A. G. Cohen and D. B. Kaplan, Phys. Lett. B470, 52 (1999) hep-th/9910132; R. Gregory, Phys. Rev. Lett. 84 (2000) 2564 hep-th/9911015; I. Olasagasti and A. Vilenkin, Phys. Rev. D62 (2000) 044014 hep-th/0003300; T. Gherghetta and M. Shaposhnikov, Phys. Rev. Lett. 85 (2000) 240 hep-th/0004014; T. Gherghetta, E. Roessl and M. Shaposhnikov, Phys. Lett. B491 (2000) 353 [hep-th/0006251]; S. Randjbar-Daemi and M. Shaposhnikov, Phys. Lett. B491 (2000) 329 [hep-th/0008087. 